Medical Center in Chicago, Illinois, reported on the findings of an investigation of the role of resistance genes and clindamycin use associated with an epidemic of $C$ difficile in four hospitals in the United States between 1989 and 1992.

Case-control studies were performed at three of the four hospitals to assess the relation of the use of clindamycin to $C$ difficile-associated diarrhea. All isolates of the epidemic strain and representative isolates of other strains identified during each outbreak were tested for susceptibility to clindamycin. Chromosomal DNA from these representative isolates was also analyzed by dot blot hybridization and amplification with the polymerase chain reaction (PCR) with the use of probes and primers from a previously described determinant of erythromycin resistance-the erythromycin ribosomal methylase B (ermB) gene-found in Clostridium perfringens and $C$ difficile.

The use of clindamyin was found to be significantly increased among patients with diarrhea due to the epidemic strain of $C$ difficile, as compared with patients whose diarrhea was due to nonepidemic strains. Exposure to other types of antibiotics or hospitalization in a surgical ward was not significantly associated with the risk of $C$ difficile-associated diarrhea due to the epidemic strain. All epidemic-strain isolates were highly resistant to clindamycin (minimal inhibitory concentration, $>256 \mu \mathrm{g} / \mathrm{mL}$ ). DNA hybridization and PCR analysis showed that all these isolates had an ermB gene, which encodes a $23 \mathrm{~S}$ ribosomal RNA methylase that mediates resistance to macrolide, lincosamide, and streptogramin antibiotics. Only $15 \%$ of the nonepidemic strains were resistant to clindamycin.

The investigators concluded that a strain of $C$ difficile that is highly resistant to clindamycin was responsible for the outbreaks of diarrhea in four hospitals in different states. The use of clindamycin is a specific risk factor for diarrhea due to this strain. Resistance to clindamycin further increases the risk of $C$ difficile-associated diarrhea, an established complication of antimicrobial use.

FROM: Johnson S, Samore MH, Farrow KA, Killgore GE, Tenover FC, Lyras D, et al. Epidemics of diarrea caused by a clindamycin-resistant strain of Clostridium difficile in four hospitals. N Engl J Med 1999;341:645-651.

\section{TB Transmission to Embalmer}

The risk of acquiring TB varies according to occupation and is high among funeral home workers. Embalmers are at particularly high risk for reactivity on tuberculin skin testing. The increased risk may be due to exposure to Mycobacterium tuberculosis during the embalming process, which involves the aspiration of blood and other body fluids from the cadaver, resulting in the generation of potentially infectious aerosols. However, the transmission of $M$ tuberculosis from a cadaver to an embalmer, with the subsequent development of active TB, has not been described.

Sterling and colleagues recently reported transmission of TB from a cadaver (case 1) to an embalmer (case 2) confirmed by DNA fingerprinting by restriction fragmentlength polymorphism (RFLP) analysis. The only known contact between the cases occurred at the time of embalming.

Case 1 was a 35-year-old man with AIDS who was hospitalized with fever and a cough. A chest radiograph showed infiltrates in the upper and middle portions of both lungs. A sputum sample was obtained for staining for acid-fast bacteria and culture, but the patient died on the day it was obtained. After the patient's death, the acid-fast smear was reported to be positive, and the culture grew $M$ tuberculosis. Blood cultures for mycobacteria were not performed.

Case 2 was a 45-year-old man who worked as an embalmer. A test for antibodies to HIV, performed 2 years before presentation, had reportedly been negative. The patient did not have a history of TB and had not undergone tuberculin skin testing. He had been an embalmer for 15 years and could not remember ever embalming a cadaver that he knew had active TB. He performed at least 300 embalmings per year, always wearing gloves and usually wearing a mask. He had no history of percutaneous exposure to blood or of chronic skin lesions. He was diagnosed with pulmonary TB 31 months after exposure to case 1.

These cases were identified as part of an ongoing study of the epidemiology of TB. DNA fingerprinting was performed with $M$ tuberculosis isolates from more than $90 \%$ of patients in Baltimore, Maryland, who had received a diagnosis of TB, confirmed by culture, between January 1994 and December 1998. Cases 1 and 2 were part of this study. RFLP analysis showed that the $M$ tuberculosis isolates from patient 1 and patient 2 had an identical 10-band fingerprint pattern.

FROM: Sterling TR, Pope DS, Bishai WR, Harrington S, Gershon RR, Chaisson RE. Transmission of Mycobacterium tuberculosis from a cadaver to an embalmer. N Engl J Med 2000;342:246-251.

\section{Epidemic Transmission of HIV in Egyptian Renal Dialysis Centers}

In 1993 an epidemic of HIV infection occurred among 39 patients at two renal dialysis centers in Egypt. El Sayad and coinvestigators from the National AIDS Programme, Ministry of Health and Population, Cairo, Egypt, and the CDC's Hospital Infections Program investigated the outbreak. The hemodialysis centers, private center A (PCA) and university center A (UCA), were visited, HIV-infected patients were interviewed, seroconversion rates at UCA were calculated, and relatedness of HIV strains was determined by sequence analysis; $34(62 \%)$ of 55 patients from UCA and $5(42 \%)$ of 12 patients from PCA were HIV-infected. The HIV seroconversion risk at UCA varied significantly with day and shift of dialysis session.

Practices that resulted in sharing of syringes among patients were observed at both centers. Unmarked syringes were reused and stored on shared trays in between use at both centers. No documentation of prescreening HIV tests for patients at the PCA was available, and the dialysis staff at this center had no trained nurses. The analyzed V3 loop sequences of the HIV strain of 12 outbreak patients were $>96 \%$ related to each other. V3 loop sequences from each of 8 